\title{
High Resolution Coherent Population Trapping on a Single Hole Spin in a Semiconductor Quantum Dot
}

\author{
Julien Houel, ${ }^{1,5}$ Jonathan H. Prechtel, ${ }^{1, *}$ Andreas V. Kuhlmann, ${ }^{1}$ Daniel Brunner, ${ }^{2}$ Christopher E. Kuklewicz, ${ }^{3}$ \\ Brian D. Gerardot, ${ }^{3}$ Nick G. Stoltz, ${ }^{4}$ Pierre M. Petroff, ${ }^{4}$ and Richard J. Warburton ${ }^{1}$ \\ ${ }^{1}$ Department of Physics, University of Basel, Klingelbergstrasse 82, CH 4056 Basel, Switzerland \\ ${ }^{2}$ Instituto de Fisica Interdisciplinar y Sistemas Complejos, IFISC (UIB-CSIC), Campus Universitat de les Illes Balears, \\ Palma de Mallorca E-07122, Spain \\ ${ }^{3}$ SUPA, Institute of Photonics and Quantum Sciences, Heriot-Watt University, Edinburgh EH14 4AS, United Kingdom \\ ${ }^{4}$ Materials Department, University of California, Santa Barbara, California 93106, USA \\ ${ }^{5}$ Institut Lumière Matière (ILM), UMR5306 Université Lyon 1/CNRS, Université de Lyon, 69622 Villeurbanne Cedex, France
}

(Received 8 July 2013; published 12 March 2014)

\begin{abstract}
We report high resolution coherent population trapping on a single hole spin in a semiconductor quantum dot. The absorption dip signifying the formation of a dark state exhibits an atomic physicslike dip width of just $10 \mathrm{MHz}$. We observe fluctuations in the absolute frequency of the absorption dip, evidence of very slow spin dephasing. We identify the cause of this process as charge noise by, first, demonstrating that the hole spin $g$ factor in this configuration (in-plane magnetic field) is strongly dependent on the vertical electric field, and second, by characterizing the charge noise through its effects on the optical transition frequency. An important conclusion is that charge noise is an important hole spin dephasing process.
\end{abstract}

DOI: 10.1103/PhysRevLett.112.107401

Coherent population trapping (CPT) is a quantum interference effect which arises in an optical $\Lambda$ system [1]. Two ground states are coupled individually by "pump" and "probe" lasers to a common upper level. At the twophoton resonance when the frequency difference of the lasers matches the frequency difference of the ground states, one of the three eigenstates has zero amplitude of the upper level, the "dark" state. CPT refers to the signature of the dark state, a dip in the probe absorption spectrum as the probe is tuned through the two-photon resonance. Specifically, for probe and pump couplings $\hbar \Omega_{1}, \hbar \Omega_{2}$ in the perturbative regime $\hbar \Omega_{1} \ll \hbar \Omega_{2} \ll \hbar \Gamma_{r}$ ( $\Gamma_{r}$ is the spontaneous emission rate from the upper state), the dip has width $\hbar \Omega_{2}^{2} / \Gamma_{r}$.

CPT is a key effect in atomic physics. First, CPT forms the microscopic basis of electromagnetically induced transparency which itself underpins a scheme for slowing light [2]. Second, the narrow dip enables the frequency separation of the ground states to be measured extremely precisely by optical means. Third, the dark state of CPT can be used for quantum control [3]. Finally, the "visibility" of the quantum interference at the CPT dip is sensitive to the ground state coherence (but insensitive to the upper state coherence) [4-7]: ground state decoherence admixes the dark state with the two bright states. The dip width sets the sensitivity to the coherence time $T_{2}$ : for $1 / T_{2} \ll \Omega_{2}^{2} / \Gamma_{r}$ the signal in the dip goes to zero but for $1 / T_{2} \gg \Omega_{2}^{2} / \Gamma_{r}$ the dip is washed out. The sensitivity to the dephasing time $T_{2}^{*}$ comes about because ground state dephasing implies a fluctuating two-photon resonance position, resulting in both an increase in dip width and a decrease in "visibility."
PACS numbers: 78.67.Hc, 03.65.Yz, 73.21.La, 78.47.-p

It is motivating to implement $\mathrm{CPT}$ in a semiconductor. Systems include excitons in GaAs quantum wells [8], bound excitons in GaAs [9], and spin states in InGaAs quantum dots [5-7]. The challenge is to engineer two ground states with $T_{2}$ and $T_{2}^{*}$ times much larger than the radiative lifetime $\tau_{r}$ of the upper state. A single electron in an InGaAs quantum dot is an obvious candidate: the spin states represent a two-level system, and spontaneous emission is fast, $\tau_{r} \sim 1 \mathrm{~ns}$ [10]. Unfortunately, in the presence of noisy nuclei, the hyperfine interaction limits $T_{2}^{*}$ to just a few ns $[11,12]$ and the CPT dip can only be observed at large optical couplings where it is inevitably broad [5]. The situation improves either by reducing the nuclear spin noise [13] or by using a quantum dot molecule [7] at a point where the first order sensitivity to spin noise vanishes.

A hole spin is potentially simpler. A heavy hole state with spin eigenvectors $J=(3 / 2), J_{z}= \pm(3 / 2)$ is predicted to become coherent in an in-plane magnetic field [14]. Conveniently, the in-plane magnetic field is exactly the field direction required to establish the $\Lambda$ system $[5,6]$. The key point is that a perfect heavy hole spin is sensitive to nuclear spin noise only along the vertical direction $z$, noise which can be suppressed by applying an external magnetic field in the $(x, y)$ plane [14]. The extent to which the idealized heavy hole picture applies to a real hole in a quantum dot has been explored in a number of recent optical experiments [6,15-21]. The Hahn echo $T_{2}$ is in the $\mu$ s range [20], a remarkable result bearing in mind the extremely limited hole spin coherence in quantum wells and bulk material $[22,23]$. Spin dephasing times $T_{2}^{*}$ lie in 
the 10-100 ns regime with significant differences from experiment to experiment [6,19-21]. CPT dips have been observed on a single hole spin [6] but these experiments used optical couplings only slightly less than the radiative decay rate, resulting in dip widths of $\sim 100 \mathrm{MHz}$. This is too large for slow light, high resolution spectroscopy and quantum metrology applications. It also renders the experiment insensitive to decoherence times above about $100 \mathrm{~ns}$.

We report here CPT on a single hole spin in the perturbative regime. A dip width of just $10 \mathrm{MHz}$ is demonstrated. The residual absorption in the center of the dip is zero (within random error), consistent with a coherence time $T_{2} \geq 1 \mu \mathrm{s}$. However, we discover a scan-toscan variation in the CPT position. We relate this to charge noise. On the one hand, we measure the dependence of the hole spin $g$ factor on vertical electric field. On the other hand, we quantify the fluctuations in vertical electric field through their effects on the optical transition, the dependence arising via the dc Stark effect. We identify charge noise as an important dephasing mechanism for the quantum dot hole spin. Charge-noise induced spin dephasing is potentially important for other spin qubits with an electric-field dependent $g$ factor and for systems with a strong spin-orbit interaction.

The semiconductor device is a $p$-type charge-tunable heterostructure to which a voltage $V_{g}$ is applied, Fig. 1(a) $[6,15,24]$. Laser spectroscopy is carried out using differential reflectivity $(\Delta R / R)$ detection, Fig. 1(b) $[25,26]$. The exciton's optical linewidth is dot dependent, typically $5-10 \mu \mathrm{eV}$ [24]. This is considerably larger than both the transform limit, $\sim 0.8 \mu \mathrm{eV}$ [27], and the linewidths on high quality $n$-type samples, $\sim 1.5 \mu \mathrm{eV}$ [25,27], and reflects additional charge noise associated with the $p$-type doping [24]. A magnetic field of $0.5 \mathrm{~T}$ is applied in the plane, and then two-color pump-probe laser spectroscopy is carried out using two coherent lasers. The experiment is very

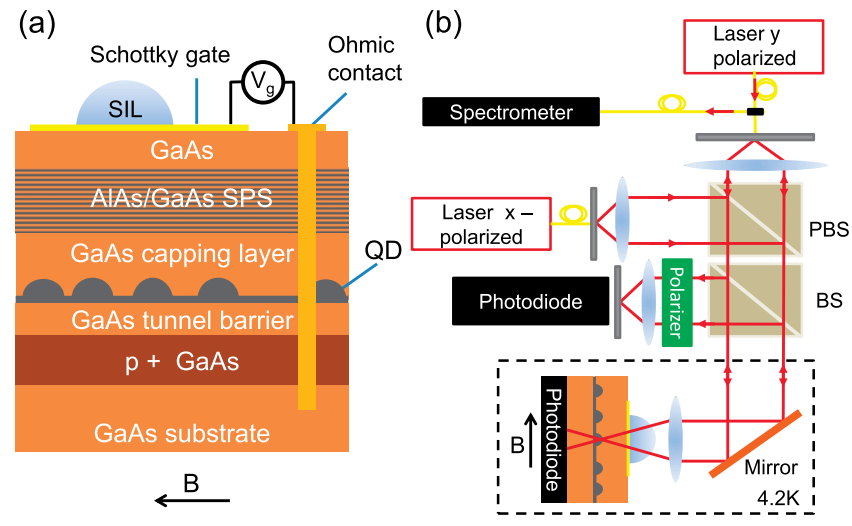

FIG. 1 (color online). (a) Layer sequence of the semiconductor heterostructure. The tunnel barrier is $25 \mathrm{~nm}$, capping layer $10 \mathrm{~nm}$ and short-period superlattice $120 \mathrm{~nm}$. Postgrowth, Ohmic contacts along with a semitransparent surface gate electrode are fabricated. (b) Schematic of the optical setup. challenging: $\Delta R / R$ is very small at the optical resonance $(0.1 \%)$; at the ultralow laser powers used here noise in the detector circuit is significant; and in the perturbative regime $\left(\hbar \Omega_{1} \ll \hbar \Omega_{2} \ll \hbar / \tau_{r}\right)$, the width of the CPT dip approaches the limit set by the mutual coherence of the lasers. We meet these challenges with a solid immersion lens to boost the $\Delta R / R$ signal, a modulation technique to reject noise in the reflectivity signal, and a stabilization scheme to lock the pump-probe frequency difference to a radio frequency reference (mutual coherence of $2.0 \mathrm{MHz}$ in $30 \mathrm{~s}$ ).

A CPT dip on a single hole spin in a magnetic field of $0.5 \mathrm{~T}$ and temperature $4.2 \mathrm{~K}$ is shown in Fig. 2. The optical couplings $\hbar \Omega_{1}$, $\hbar \Omega_{2}$ were determined by measuring an Autler-Townes splitting at high laser powers, extrapolating the couplings to low laser powers using the scaling $\hbar \Omega \propto$ $\sqrt{P}$ ( $P$ is the laser power), Fig. 3(a). In the CPT experiment, $\hbar \Omega_{2}$ is a factor of 3 lower than the spontaneous decay rate $\Gamma_{r}=1 / \tau_{r}$. The full-width-at-half-maximum of the CPT dip is just $13 \mathrm{MHz}$, equivalently $54 \mathrm{neV}$, corresponding to just $10^{-7}$ of the frequency of the optical transitions, and just $10^{-3}$ of the thermal energy. Figure 2 constitutes our main result: a CPT dip linewidth in the $\mathrm{MHz}$ regime, a spectral sensitivity usually associated with atomic physics and not a semiconductor experiment.

The solid lines in Figs. 2 and 3(a) correspond to the results of a 3-level density matrix $(\rho)$ calculation [6]. The result for $\rho_{13}$ [2] is convoluted with a Lorentzian distribution of width $\Gamma_{X}$ in the energy of the upper level $E_{3}$ in order to describe the effects of charge noise. $\Gamma_{X}$ is known from the one-laser characterization at $B=0$. The result is then convoluted again, this time with a Lorentzian function of

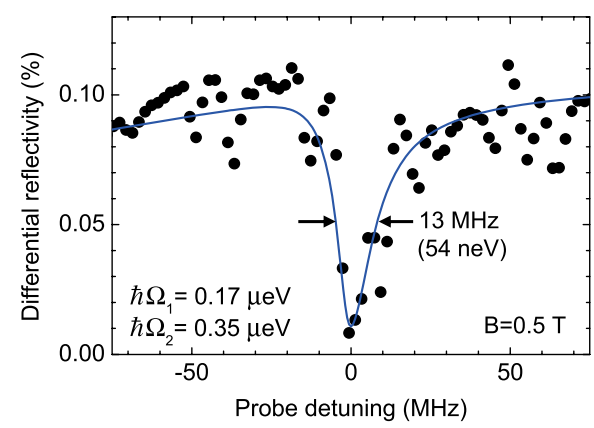

FIG. 2 (color online). Probe absorption in the presence of a close-to-resonant pump laser on a single InGaAs quantum dot containing a single hole spin at $B=0.5 \mathrm{~T}$ and $T=4.2 \mathrm{~K}, 10 \mathrm{~s}$ integration per point. The absorption (here, differential reflectivity $\Delta R / R$ ) shows an absorption dip signifying coherent population trapping. The solid line shows the result of a 3-level density matrix model $\left(\hbar \Omega_{1}=0.17, \hbar \Omega_{2}=0.35, \hbar \delta_{2}=-2.5 \mu \mathrm{eV}\right.$, $\hbar / \tau_{r}=0.8 \mu \mathrm{eV}, T_{2} \geq 1 \mu \mathrm{s}, T_{1} \gg T_{2}$ ) convoluted with a Lorentzian with FWHM $\Gamma_{X}=5 \mu \mathrm{eV}(1.22 \mathrm{GHz})$ to describe slow exciton dephasing, and then with a Lorentzian with FWHM $8.3 \mathrm{neV}(2.0 \mathrm{MHz})$ to account for the mutual coherence of the lasers. 
(a)

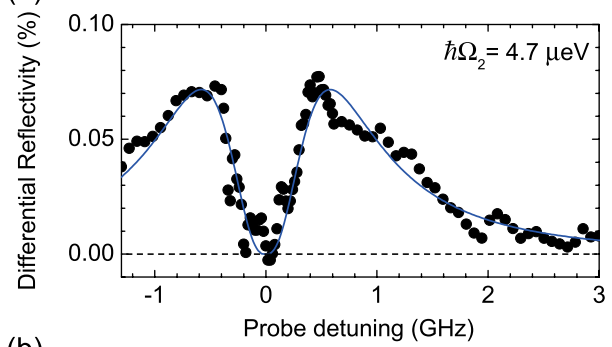

(b)

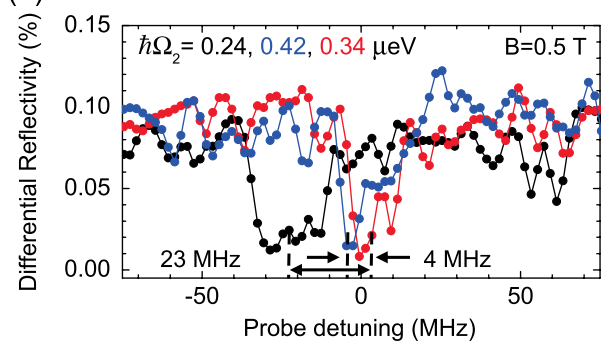

FIG. 3 (color online). (a) Probe absorption versus probe detuning on the same quantum dot as Fig. 2 in the regime $\hbar \Omega_{2} \gg$ $\hbar / \tau_{r}$ showing an Autler-Townes splitting. The solid curve is a fit to the data, $\hbar \Omega_{2}=4.7 \mu \mathrm{eV}, \hbar \delta_{2}=0.0 \mu \mathrm{eV}$, as fit parameters. $\Gamma_{X}=7.5 \mu \mathrm{eV}$ is taken from $\Delta R / R$ at $B=0$. (b) Probe absorption versus probe detuning on the same quantum dot in the regime $\hbar \Omega_{2} \ll \hbar / \tau_{r}$. Three curves are shown under close-to-identical conditions showing a shift in the location of the dip in one case by $4 \mathrm{MHz}(17 \mathrm{neV})$, and in another case by $23 \mathrm{MHz}(95 \mathrm{neV})$.

width $2.0 \mathrm{MHz}(8.3 \mathrm{neV})$ to describe the limited mutual coherence of the lasers. In the limit of large $\hbar \Omega_{2}$, the Autler-Townes experiment, $\hbar \Omega_{2}$ is treated as a fit parameter, and the result describes the absorption envelope extremely well, Fig. 3(a). In the limit of small $\hbar \Omega_{2}$, the CPT experiment, the result describes the dip width and depth extremely well (Fig. 2). In the CPT limit, there are no unknowns apart from a small uncertainty in $\hbar \delta_{2}$, the pump detuning (see below).

The residual signal in the CPT dip of Fig. 2 can be fully accounted for by the mutual coherence of the lasers: the condition $1 / T_{2} \ll \Omega_{2}^{2} / \Gamma_{r}$ is satisfied, which translates to $T_{2} \geq 1 \mu \mathrm{s}$. Furthermore, the width of the CPT dip in Fig. 2 is described with the three-level model without ensemble averaging. This sets a lower limit on the dephasing time: from the uncertainty in the CPT width (Fig. 2) we determine $T_{2}^{*} \geq 100 \mathrm{~ns}$. A measurement of $T_{2}^{*}$ on a single emitter involves an integration of the experiment in time, equivalently over a frequency bandwidth. The value of $T_{2}^{*}$ deduced from Fig. 2 represents an integration over measurement frequencies starting at about $0.01 \mathrm{~Hz}$.

We take advantage of the narrow dip to perform high resolution dark state spectroscopy. We find that the frequency of the dip fluctuates from scan to scan, Fig. 3(b), over a range of $\sim 5 \mathrm{MHz}(\sim 20 \mathrm{neV})$. Occasionally, larger frequency shifts are observed, possibly with an unusual line shape, Fig. 3(b). These effects point to the presence of very slow fluctuations in the frequency separation of the hole spin ground states. In terms of $T_{2}^{*}$, we find that $T_{2}^{*}$ falls to just $\sim 30 \mathrm{~ns}$ when the measurement bandwidth starts at $\sim 0.0001 \mathrm{~Hz}$. It is unlikely that nuclear spin noise is responsible for this extra noise at very low frequencies. On the one hand, nuclear spin noise lies at higher frequencies [27]; on the other hand, we have not observed any hysteresis effects in the CPT experiment, the typical signature of the nuclear spins. The slow CPT fluctuations are reminiscent of the low frequency wanderings of the optical transition which has a $1 / f$-like noise spectrum and arises from charge noise [27]. We therefore look for a link between charge noise and the hole $g$ factor.

We have characterized the eigenenergies as a function of $V_{g}$ by measuring the photoluminescence (PL) from the positively charged trion $X^{1+}$ in an in-plane magnetic field $B=9 \mathrm{~T}$, Fig. 4(a). We resolve 4 lines corresponding to the two "vertical" transitions and the two "diagonal" transitions, Figs. 4(a) and 4(b). Figure 4 shows the $V_{g}$
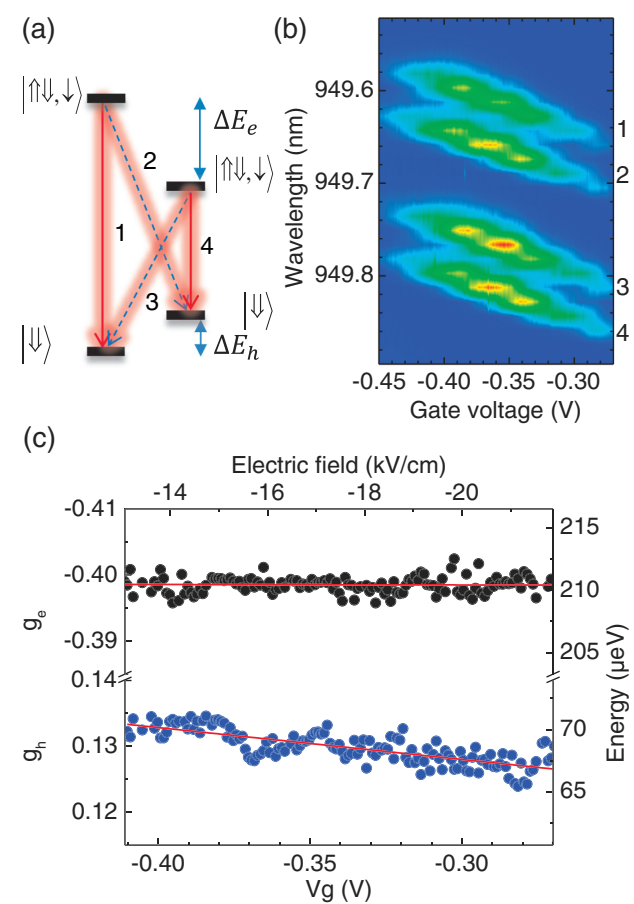

FIG. 4 (color online). (a) The quantum states of a single hole spin in an in-plane magnetic field. $\uparrow, \downarrow$ denotes an electron spin, $\Uparrow, \Downarrow$ a hole spin. (b) Photoluminescence on a single InGaAs quantum dot (different quantum dot from Figs. 2 and 3 but from the same wafer) at $B=9.0 \mathrm{~T}$ and $T=4.2 \mathrm{~K}$ as a function of $V_{g}$ over the extent of the single hole Coulomb blockade plateau. Four transitions are visible, labeled $1-4$, and identified in (a). The width of each peak is determined by the spectrometer detector (system resolution $50 \mu \mathrm{eV}$ ). The apparent steps are an artifact arising from the pixelated detector. (c) Electron and hole $g$ factors, $g_{e}$ and $g_{h}$, versus $V_{g}$. $V_{g}$ is converted into vertical electric field $F$ with $F=-e\left(V_{g}+V_{o}\right) / D$ with $D=155 \mathrm{~nm}$ and Schottky barrier $V_{o}=0.62 \mathrm{~V}$. Under the assumption of a negative $g_{e}, g_{h}$ is positive. The error on each point is $\sim 2 \mu \mathrm{eV}$ using a routine which corrects for the detector pixelation. 
dependence of the electron and hole Zeeman energies. To within error, $\sim 0.25 \%$, the electron Zeeman energy $E_{Z}^{e}$ is independent of $V_{g}$; in contrast, the hole Zeeman energy $E_{Z}^{h}$ changes by $\sim 5 \%$ over the $X^{1+}$ plateau. Defining the $g_{h}$ via $E_{Z}^{h}=g_{h} \mu_{B} B$, we find $g_{h}=0.15+\alpha F$ with $\alpha=$ $8.6 \times 10^{-4} \mathrm{~cm} / \mathrm{kV}$ ( $F$ is the vertical electric field).

The dependence of $g_{h}$ on $F$ creates a mechanism by which charge noise can result in spin dephasing: electric field fluctuations cause changes to the hole spin precession frequency. In particular, the low frequency charge noise causes both the optical transition energy and the CPT dip position to wander. Specifically, the charge noise in a bandwidth $f_{\text {scan }} \leq f \leq 1 / \tau_{r}$ can be determined by measuring the optical linewidth at rate $f_{\text {scan }}$, converting the inhomogeneous broadening into electric field noise via the known Stark shift. This is easy to measure with the resonant laser spectroscopy employed here. Charge noise at lower frequencies $f \leq f_{\text {scan }}$ results in scan-to-scan fluctuations of the resonance energy. CPT is recorded on the same quantum dot experiencing the same noise: a powerful connection can therefore be made between the optical linewidth and the CPT experiment.

The optical linewidth in Fig. 2 is $5.0 \mu \mathrm{eV}$. Together with the known Stark shifts, $d E / d F=18 \mu \mathrm{eV} / \mathrm{kV} \mathrm{cm}^{-1}$ $\left[d E / d V_{g}=1.12 \mu \mathrm{eV} / \mathrm{mV}\right]$, this results in $\Delta F=$ $0.28 \mathrm{kV} \mathrm{cm}^{-1}\left[\Delta V_{g}=4.5 \mathrm{mV}\right]$. This charge noise $\Delta F$ results in turn in a fluctuation in $g_{h}$ of $\Delta g_{h}=2.4 \times 10^{-4}$, equivalently, $\Delta E_{Z}^{h}=7 \mathrm{neV}(1.7 \mathrm{MHz})$ at $0.5 \mathrm{~T}$. This broadening is comparable to the frequency resolution of the experiment and therefore plays a small role. This explains why the CPT dip in Fig. 2 can be explained without taking into account charge noise-induced dephasing. The effects in Fig. 3(b) arise when $f_{\text {scan }}$ is reduced even further. In this case, ultralow frequency flicker noise results in wanderings of the quantum dot optical resonance typically by one or two linewidths over the course of many minutes [27]. These optical shifts induce in turn wanderings of the CPT dip, by a few MHz, as observed in Fig. 3(b). The larger shifts in CPT position, Fig. 3(b), may represent highly unlikely but more extreme changes to the electrostatic environment of the quantum dot.

Our results point to the role of charge noise in dephasing a quantum dot hole spin. In this experiment, the charge noise in $E_{Z}^{h}$ implies a dephasing time of $T_{2}^{*, c} \simeq 100 \mathrm{~ns}$ on integrating noise in a bandwidth starting around $0.01 \mathrm{~Hz}$ at $B=0.5$ T. $T_{2}^{*, c}$ reflects an upper limit to $T_{2}^{*}$ for a given $f_{\text {scan }}$ : other processes could reduce $T_{2}^{*}$ further. A consistency check is that the measured $T_{2}^{*}$ in a similar bandwidth (Fig. 2) cannot be higher than $T_{2}^{*, c}$. This is indeed the case. Specifically, the experiment demonstrates that charge noise is the dominant dephasing mechanism at very low values of $f_{\text {scan }}$ [Fig. 3(b)].

The key to increasing $T_{2}^{*}$ is to reduce the charge noise. Some quantum dots in the sample have lower optical linewidths despite similar Stark shifts pointing to a lower level of local charge noise and for these quantum dots we can expect $T_{2}^{*} \geq 100 \mathrm{~ns}$. Generally speaking, these resonant laser spectroscopy experiments have low charge noise, much less than in experiments with nonresonant excitation [27]. Experiments with more charge noise will therefore give smaller hole spin $T_{2}^{*}$ values. Charge noise also results in a $B$ dependence of $T_{2}^{*}$. We find that $d g_{h} / d F$ is $B$ independent: the fluctuations in $E_{Z}^{h}$ increase linearly with increasing $B$ for constant charge noise implying that $T_{2}^{*}$ scales as $1 / B$ : $T_{2}^{*} \simeq \hbar /\left(\left|d g_{h} / d F\right| \Delta F \mu_{B} B\right)$. The $B$ dependence may be obscured should other dephasing mechanisms come into play at higher magnetic fields, for instance hyperfine coupling $[20,21]$ or the interaction with phonons [16,28]. We note, however, that hole spin dynamics at magnetic fields of several Tesla reveal smaller $T_{2}^{*}$ values $[20,21]$ than those at low magnetic field $[6,19]$, and this is consistent with charge noise-dominated spin dephasing. We stress that an advantage of the present experiment is that the charge noise is measured in situ via the laser spectroscopy.

In conclusion, we report $10 \mathrm{MHz}$ wide CPT dips in laser spectroscopy experiments on a quantum dot hole spin. The quantum dot is embedded in a very good but imperfect device. Charge noise causes slow wanderings of the CPT dip. There are a number of mitigating strategies. First, $p$ type devices need to be developed with less charge noise, ideally with the low levels of charge noise associated with the best $n$-type devices. Second, the dependence of the hole $g$ factor on electric field, while possibly an attractive feature for electrical qubit control, can be reduced by appropriate quantum dot design.

We acknowledge financial support from NCCR QSIT, EU ITN $S^{3}$ NANO, Royal Society, and EPSRC.

*jonathan.prechtel@unibas.ch; http://nano-photonics.unibas.ch/

[1] E. Arimondo, Prog. Opt. 35, 257 (1996).

[2] M. Fleischhauer, A. Imamoglu, and J. P. Marangos, Rev. Mod. Phys. 77, 633 (2005).

[3] K. Bergmann, H. Theuer, and B. W. Shore, Rev. Mod. Phys. 70, 1003 (1998).

[4] A. Imamoglu, Phys. Status Solidi B 243, 3725 (2006).

[5] X. Xu, B. Sun, P. R. Berman, D. G. Steel, Allan, Bracker, D. Gammon, and L. J. Sham, Nat. Phys. 4, 692 (2008).

[6] D. Brunner, B. D. Gerardot, P. A. Dalgarno, G. Wüst, K. Karrai, N. G. Stoltz, P. M. Petroff, and R. J. Warburton, Science 325, 70 (2009).

[7] K. M. Weiss, J. M. Elzerman, Y. L. Delley, J. MiguelSanchez, and A. Imamoğlu, Phys. Rev. Lett. 109, 107401 (2012).

[8] M. C. Phillips, H. Wang, I. Rumyantsev, N. H. Kwong, R. Takayama, and R. Binder, Phys. Rev. Lett. 91, 183602 (2003).

[9] M. Sladkov, A. U. Chaubal, M. P. Bakker, A. R. Onur, D. Reuter, A. D. Wieck, and C. H. van der Wal, Phys. Rev. B 82, 121308 (2010). 
[10] P. A. Dalgarno, M. Ediger, B. D. Gerardot, J. M. Smith, S. Seidl, M. Kroner, K. Karrai, P. M. Petroff, A. O. Govorov, and R. J. Warburton, Phys. Rev. Lett. 100, 176801 (2008).

[11] I. A. Merkulov, A. L. Efros, and M. Rosen, Phys. Rev. B 65, 205309 (2002).

[12] A. V. Khaetskii, D. Loss, and L. Glazman, Phys. Rev. Lett. 88, 186802 (2002).

[13] X. Xu, W. Yao, B. Sun, D. G. Steel, A. S. Bracker, D. Gammon, and L. J. Sham, Nature (London) 459, 1105 (2009).

[14] J. Fischer, W. A. Coish, D. V. Bulaev, and D. Loss, Phys. Rev. B 78, 155329 (2008).

[15] B. D. Gerardot, D. Brunner, P. A. Dalgarno, P. Ohberg, S. Seidl, M. Kroner, K. Karrai, N. G. Stoltz, P. M. Petroff, and R. J. Warburton, Nature (London) 451, 441 (2008).

[16] D. Heiss, V. Jovanov, M. Bichler, G. Abstreiter, and J. J. Finley, Phys. Rev. B 77, 235442 (2008).

[17] A. J. Ramsay, S. J. Boyle, R. S. Kolodka, J. B. B. Oliveira, J. Skiba-Szymanska, H. Y. Liu, M. Hopkinson, A. M. Fox, and M. S. Skolnick, Phys. Rev. Lett. 100, 197401 (2008).

[18] T. M. Godden, S. J. Boyle, A. J. Ramsay, A. M. Fox, and M. S. Skolnick, Appl. Phys. Lett. 97, 061113 (2010).

[19] S. A. Crooker, J. Brandt, C. Sandfort, A. Greilich, D. R. Yakovlev, D. Reuter, A. D. Wieck, and M. Bayer, Phys. Rev. Lett. 104, 036601 (2010).
[20] K. De Greve, P. L. McMahon, D. Press, T. D. Ladd, D. Bisping, C. Schneider, M. Kamp, L. Worschech, S. Hofling, A. Forchel, and Y. Yamamoto, Nat. Phys. 7, 872 (2011).

[21] A. Greilich, S. G. Carter, D. Kim, A. S. Bracker, and D. Gammon, Nat. Photonics 5, 702 (2011).

[22] T. C. Damen, L. Vina, J. E. Cunningham, J. Shah, and L. J. Sham, Phys. Rev. Lett. 67, 3432 (1991).

[23] X. Marie, T. Amand, P. Le Jeune, M. Paillard, P. Renucci, L. E. Golub, V. D. Dymnikov, and E. L. Ivchenko, Phys. Rev. B 60, 5811 (1999).

[24] B. D. Gerardot, R. J. Barbour, D. Brunner, P. A. Dalgarno, A. Badolato, N. Stoltz, P. M. Petroff, J. Houel, and R. J. Warburton, Appl. Phys. Lett. 99, 243112 (2011).

[25] A. Högele, S. Seidl, M. Kroner, K. Karrai, R. J. Warburton, B. D. Gerardot, and P. M. Petroff, Phys. Rev. Lett. 93, 217401 (2004).

[26] B. Alén, A. Högele, M. Kroner, S. Seidl, K. Karrai, R. J. Warburton, A. Badolato, G. Medeiros-Ribeiro, and P. M. Petroff, Appl. Phys. Lett., 89, 123124 (2006).

[27] A. V. Kuhlmann, J. Houel, A. Ludwig, L. Greuter, D. Reuter, A. D. Wieck, M. Poggio, and R. J. Warburton, Nat. Phys. 9, 570 (2013).

[28] M. Trif, P. Simon, and D. Loss, Phys. Rev. Lett. 103, 106601 (2009). 